OPEN

SUBJECT AREAS:

CHEMICAL

ENGINEERING

NANOPARTICLES

Received

7 October 2014

Accepted

18 December 2014

Published

27 January 2015

Correspondence and requests for materials should be addressed to S.J.L. (sjlee@postech. ac.kr)

\section{Optimum periodicity of repeated contractile actions applied in mass transport}

\author{
Sungsook Ahn ${ }^{1,2} \&$ Sang Joon Lee ${ }^{1,2}$
}

\begin{abstract}
'Biofluid and Biomimic Research Center, Pohang University of Science and Technology, Pohang, 790-784, Korea, ${ }^{2}$ Department of Mechanical Engineering, Pohang University of Science and Technology, Pohang, 790-784, Korea.
\end{abstract}

Dynamically repeated periodic patterns are abundant in natural and artificial systems, such as tides, heart beats, stock prices, and the like. The characteristic repeatability and periodicity are expected to be optimized in effective system-specific functions. In this study, such optimum periodicity is experimentally evaluated in terms of effective mass transport using one-valve and multi-valve systems working in contractile fluid flows. A set of nanoscale gating functions is utilized, operating in nanocomposite networks through which permeates selectively pass under characteristic contractile actions. Optimized contractile periodicity exists for effective energy impartment to flow in a one-valve system. In the sequential contractile actions for a multi-valve system, synchronization with the fluid flow is critical for effective mass transport. This study provides fundamental understanding on the various repeated periodic patterns and dynamic repeatability occurring in nature and mechanical systems, which are useful for broad applications.

$\mathrm{P}$ eriodically repeated patterns are pervasive in natural and artificial systems where characteristic spatial or temporal information can be determined. Gravitational forces cause tides in the ocean and Earth with diurnal and semidiurnal periods ${ }^{1}$. Earthquakes in a specific region are anticipated by repeated oscillation patterns ${ }^{2}$. Among the biological periodic processes, the rhythmic process is a central function of life, such as limb motions in walking, heart beating, metabolism, growth, hormone regulation, and circadian clocks ${ }^{3,4}$. In this context, pulsatile pressure and dynamic flow in arteries have attracted increasing interest to explain diseaserelated hemodynamic phenomena ${ }^{5}$.

To investigate the physiological dynamic phenomena occurring in nature, in vitro model systems have been usefully employed. In many model systems, the water-based fluid flows through rigid pipes have been handled. However, these studies have technical limitation in their plausible adoptability for actual dynamics. Selective mass transport with controllable flexibility ${ }^{6}$ thus has a great advantage in mimicking the real dynamics actually occurring in many biological systems. For example, human skin, a typical natural responsive membrane, acts as a permeable multifunctional membrane which interacts and responds to the surrounding environments such as light, heat, cold, humidity, chemicals, and mechanical stress ${ }^{7}$. Responsive materials have a great advantages in broad application areas such as controlled-release agents ${ }^{8-9}$, responsive coatings ${ }^{10}$ and artificial organs ${ }^{11}$. In addition to the molecular separation by size and charge for which traditional membranes are employed, responsive membranes are designed to dynamically respond to the varying environments. Although this dynamic aspect is essential in the behaviors of responsive materials, it has not been thoroughly investigated because suitable model systems are lacking.

Unlike energy transfer mechanisms (e.g., tides and earthquakes) that employ Fourier's law, mass transport is mainly governed by conventional physical laws including acceleration and gravitational rules. In this study, dynamic mass transport through stimuli responsive soft condensed matters (nanocomposites) is investigated as a new model system to study the dynamically repeated patterns occurring in contractile fluid flows. By changing the dynamic factors, the output patterns of permeate molecules are systematically modulated. Based on the obtained results, we propose a certain optimized pattern for periodic control in dynamic mass transport. This study is the first systematic investigation in which periodic repeatability and effective mass transport are conceptually combined. The obtained results would be useful for understanding various periodic patterns and dynamic mechanics which are abundant in various natural and artificial systems. 


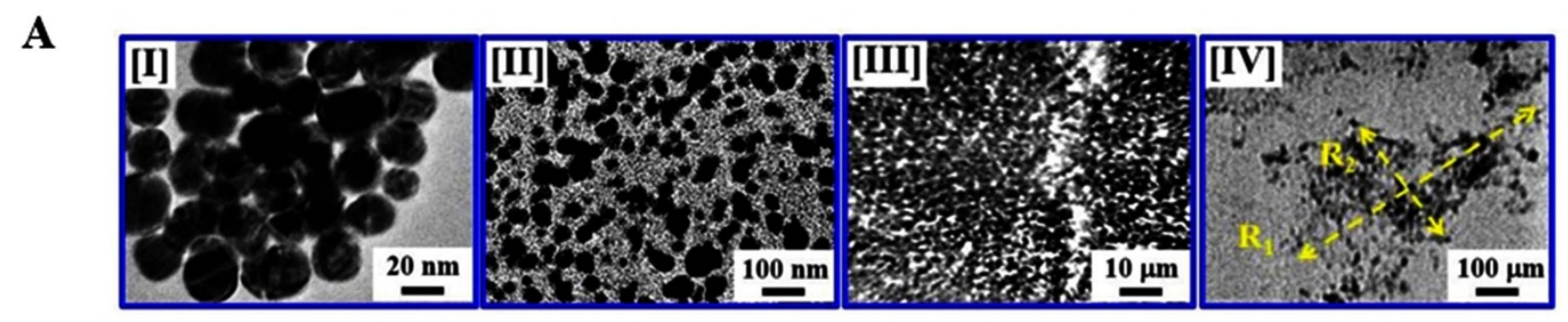

B

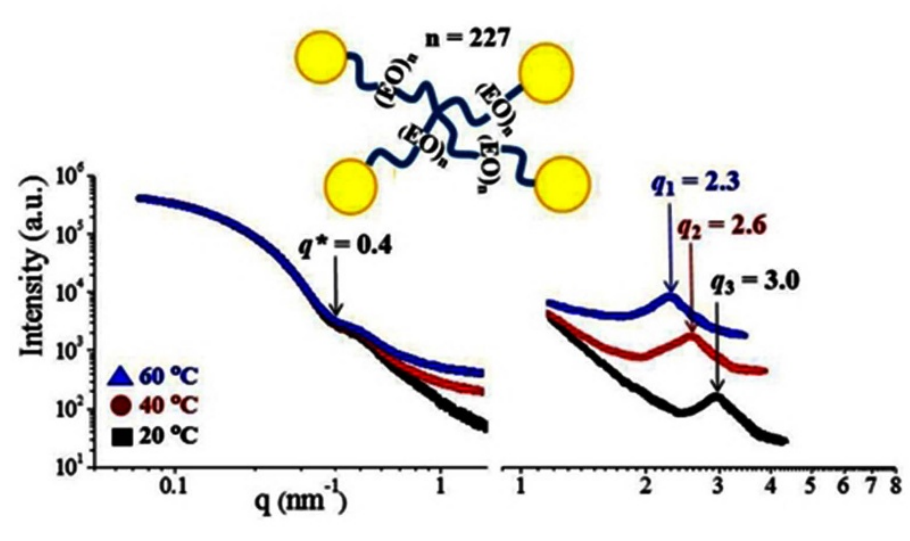

C
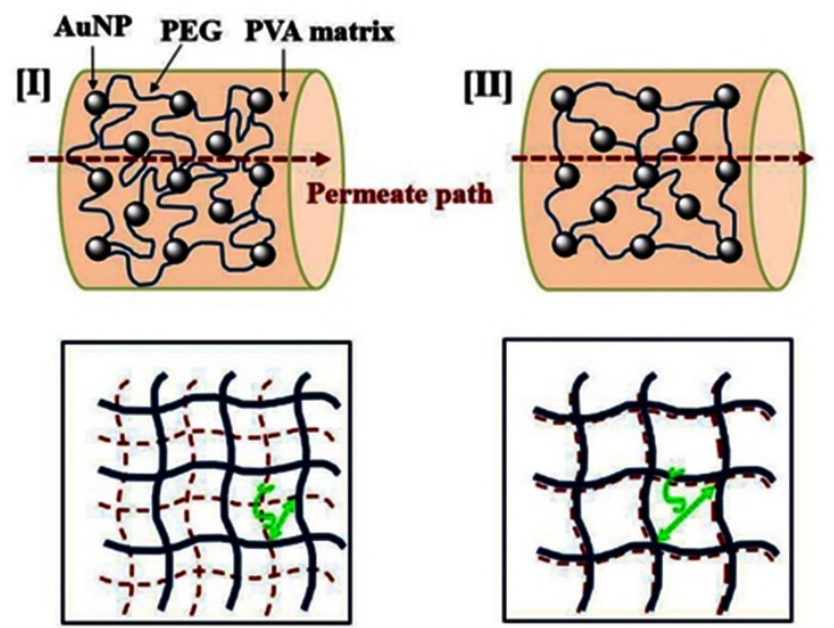

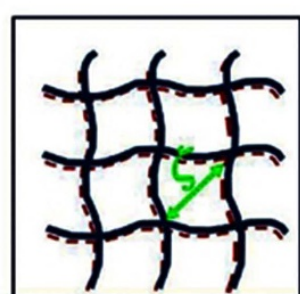

Figure $1 \mid$ (a) [I] TEM image of gold nanoparticle (AuNP) use for linterlinking. Binary functional polyethylene oxide (PEO) having EO unit number of $n$ $=227$ (2PEG 10000) is used for linking AuNPs. Representative images of the designed nanocomposites. [II] TEM, [III] X-ray nanoscopy (XN) and [IV] X-ray microscopy (XM). (b) Small angle X-ray scattering (SAXS) results of the designed AuNP-PEG nanocomposite in solution state in broad $q$ ranges (two Sample-to-detector distance conditions are combined). A critical $q$ value (marked by $q^{*}$ ) exist from which temperature-responsiveness is diversified, indicating dual regions of the designed network: stable large-scale domain at low $q$ and responsive small-scale domain at high $q$. Characteristic size is determined according to the temperature detected by $q_{1}\left(60^{\circ} \mathrm{C}\right), q_{2}\left(40^{\circ} \mathrm{C}\right)$ and $q_{3}\left(20^{\circ} \mathrm{C}\right)$. (c) Illustration of responsive network structures. Pore size variation of the nanocomposites in PVA matrix induced by the stimuli-responsive PEOs through which permeates are transported. [I] Swollen PEOs generates (or block) small path for permeate molecules. [II] Shrunken PEOs allow relatively wide pathway for effective permeate transport. The correlation lengths $(\zeta)$ are determined based on the pictures below. Bold lines denote stable connection, whereas dotted lines indicates flexibly responsive chains by the external stimuli.

\section{Results}

Temperature-responsive nanocomposite. Citrate-stabilized colloidal gold nanoparticles (AuNPs) are prepared in aqueous solution. The concentration is adjusted to be around $2.4 \times 10^{12}$ AuNPs $/ \mathrm{mL}$ (Supporting Information) ${ }^{12-13}$. The transmission electron microscopy (TEM) mage in Figure 1a[I] confirms the average diameter of the prepared single AuNP is $20 \mathrm{~nm}$ diameter. Binary thiol end-capped functional polyethylene oxide (PEO) are incorporated for AuNP interconnection. The molecular weight between the junctions is adjusted to be $\mathrm{M}_{\mathrm{p}}=10,000\left((\mathrm{EO})_{\mathrm{n}}, \mathrm{n}=227\right)$. The TEM image in Figure $1 \mathrm{a}[\mathrm{II}]$ shows that the average size of the clusters in nanometerscale $^{14-15}$. Considering the multi-reactive sites on a single AuNP, the number of incorporated PEO molecules is controlled to $\times 10, \times 50$, and $\times 100$ times that of AuNPs (Supporting Information). A network is hardly formed if binary PEO monomers are only activated. However, highly interconnected composite clusters are formed as a result of multiple reactions of AuNP surface to thiol groups at the ends of the PEO. Multiple PEO linkages are attached to the surface of an AuNP to form networked assemblies. They also possess additional levels of complexity and anisotropy that can be exploited in self-assembly. The crosslink density $(\rho)$ of the fully-linked network is inversely proportional to the molecular weight between the junction points $\left(\mathrm{M}_{\mathrm{p}}\right)$.

Nano-scale images are obtained at the 7C X-ray nanoimaging (XNI) beamline at Pohang Accelerator Laboratory (PAL, Pohang,
Korea) using high X-ray absorption coefficient of $\mathrm{Au}^{13}$ (Supporting Information) (Figure 1a[III]). Beam size is adjusted to $100 \mu \mathrm{m} \times$ $100 \mu \mathrm{m}$ at $6.7 \mathrm{keV}$ energy. Spatial resolution is approximately $100 \mathrm{~nm}$. A texture of a composite cluster is prominent as black dots because of embedded AuNPs of high X-ray absorption. In Figure $1 \mathrm{a}[\mathrm{IV}]$, the X-ray micro imaging results obtained at the $6 \mathrm{D} \mathrm{X}$-ray Micro Imaging (XMI) beamline at PAL provide a larger field-of-view than XNI (Supporting Information). Its spatial resolution is approximately $4 \mu \mathrm{m}$ at the sample-to-detector distance of $30 \mathrm{~cm}$ and FOV is adjusted to $1200 \mu \mathrm{m} \times 900 \mu \mathrm{m}$. Discrete clusters are detected by $\mathrm{XMI}$ and the size of the cluster is evaluated. Assuming that the cluster is in an ellipsoidal shape, the longer length $R_{1}$ is determined on a cluster and then the shorter $\mathrm{R}_{2}$ is evaluated at the center point of the $\mathrm{R}_{1}$ at a perpendicular direction. Arithmetically averaged cluster sizes from 10 clusters are summarized using standard deviation (Fig. S1). Increased PEO to AuNP values from $\times 10, \times 50$ to $\times 100$ (PEG/ AuNP) can enhance the size of the clusters.

Figure $1 \mathrm{~b}$ presents the small-angle X-ray scattering (SAXS) results conducted at the $4 \mathrm{C}$ beamline at the PAL with the designed nanocomposite networks in aqueous solution. Depending on the molecular weight and architecture, aqueous PEO solutions exhibit unique physical property: the solubility decrease and/or phase separation occurs above the critical temperature (lower critical solution temperature, LCST $)^{16-17}$. At a fixed PEG concentration $(100 \mathrm{mmol} / \mathrm{L})$, tem- 
perature-responsiveness of the designed nanocomposites is compared at three different temperature conditions $\left(20,40\right.$ and $\left.60^{\circ} \mathrm{C}\right)$. A characteristic $q$ value $\left(q^{*}=0.4 \mathrm{~nm}^{-1}\right)$ indicates that the solubility of the nanocomposite network decreases according to the increase in temperature from 20 to $60^{\circ} \mathrm{C}$, exhibiting the characteristic LCST. As temperature increases further, it moves to a lower $q$ region, resulting in the formation of large structures. However, the $q^{*}$ point moves to a higher $q$ region as the incorporated PEO amount increases (Figure S2) The organic-inorganic hybrid composites exhibit deviation from the typical elasticity of rubber, due to characteristic contribution of individual components. The designed nanocomposite networks are composed of dual domains, different from typical responsive materials of isotropic scale changes. The X-ray scattering intensity $\mathrm{I}(q)$ is experimentally determined as a function of the scattering vector $q$ whose modulus is given by $q=(4 \pi / \lambda) \sin (\theta / 2)$, where $\lambda$ is the X-ray wavelength and $\theta$ is the scattering angle. Since our composites are microscopically isotropic the intensities will depend only on the modulus of $q$ that for SAXS is given by $q \approx(2 \pi / \lambda) \theta$. From the obtained peaks at the SAXS profile, the size-definable structures marked by $q_{1}=2.3 \mathrm{~nm}^{-1}, q_{2}=2.6 \mathrm{~nm}^{-1}$, and $q_{3}=$ $3.0 \mathrm{~nm}^{-1}$ are generated at 60,40 and $20^{\circ} \mathrm{C}$, exhibiting larger size formation at increased temperature conditions.

Based on the obtained SAXS results, temperature-responsive nano-scale networks are suggested as illustrated in Figure 1c (left). The correlation length $(\zeta)$, the distance between the junction points of the network, changes according to temperature. Compared with the lower temperature condition of highly swollen state [I], the $\zeta$ values increase due to the shrinkage at an increased temperature [II]. The selected permeates pass the nanocomposites more effectively through larger $\zeta$ [II] with increasing temperature than through smaller $\zeta$ at a lower temperature [I]. The designed nanocomposites are embedded in poly(vinyl alcohol) (PVA) gel matrix (3wt\%, $\left.\mathrm{M}_{\mathrm{w}} \sim 72,000 \mathrm{Merck}\right)$, keeping the temperature-responsiveness of nanocomposites without leakage and shape deformation ${ }^{18}$. PVA matrix is ideal as a nanocomposite matrix, because no significant swelling or shrinking occurs under the designed experimental conditions. As the temperature increases several times above and below the transition point, the release of nanocomposites releasing from the PVA matrix is not detected. The designed nanocomposites in PVA matrix exhibits differentiated paths for the selected permeates according to the temperature condition ${ }^{5}$.

Controlled mass transport through responsive nanocomposites. Mass transport is effectively controlled using the designed apparatus (Fig. 2a). Selected permeate molecules ${ }^{19-20}$ pass through the nanocomposites loaded in a designed diffusion cell. The outlet of permeates is a function of permeate input frequency $\left(f_{\text {in }}\right)$, inlet flow rate $(r)$, and geometry of nanocomposite controlled by temperature $(\mathrm{T})$. The concentration-controlled permeate solutions are loaded at a designed time interval to generate periodic input. Approximately $0.01 \mu \mathrm{L}$ of $0.1 \mathrm{~g} / \mathrm{mL}$ rhodamine $6 \mathrm{G}$ aqueous solution is loaded for one shot, and the number of shots in a minute is varied by changing the $f_{\text {in }}$ from $1 \mathrm{~min}^{-1}$ to $8 \mathrm{~min}^{-1}$. The signal from the eluted permeates is recorded in a continuous mode (see Methods). The nanocomposite is embedded in the PVA matrix for molding into a cylinder (diameter: $0.1 \mathrm{~cm}$; length: $10 \mathrm{~cm}$ ). This cylinder-shaped nanocomposite pellet is loaded into a humiditycontrolled $0.1 \mathrm{~cm}$-thick glass tube. The tube has heating coils, and it is connected to the inlet pressure and signal detecting devices. The durability and repeatability of the nanocomposite are verified up to $500 \mathrm{~min}$ of successive mechanical repetitions. Thus, all the experiments in the previous study are performed under this condition (Supporting Information).

The time for the permeate outlet is normalized to eliminate the condition-specific $R_{t}$. Therefore, the time is expressed as $\Delta t=t-t_{0}$ in the graph, where the zero point $\left(t_{0}\right)$ is the time at which injected permeates are first detected as an outlet. At the increased temperature condition of $60^{\circ} \mathrm{C}$, the permeate is released in the detector (on position), and the permeate release is stopped at $20^{\circ} \mathrm{C}$ (off position). The temperature-controlled part in the tube is compartmented using individual heating coils to generate spatio-temporal modulation. Thus the structures can be regionally controlled by artificial gating functions. The input and output signals are highly synchronized as shown in Figure $2 b$. The left graph is the temperature signal, while the right graph exhibits detection signal of the outlet. The interval of the input signal is controlled to be longer than $15 \mathrm{~s}$ to provide sufficient time for the responsiveness of the nanocomposite. With carefully controlled experimental conditions, the results exhibit sharp synchronization responding to the applied temperature stimuli.

At the inlet flow rate $(r)$ of $10 \mu \mathrm{L} / \mathrm{min}\left(10^{-2} \mathrm{~cm}^{3} / \mathrm{min}\right)$, proper repetition signals of rhodamine $6 \mathrm{G}$ are obtained for a periodic pattern beyond the characteristic retention time $\left(\mathrm{R}_{\mathrm{t}}\right)$ during which permeates are retained inside the diffusion cell (Figure $2 \mathrm{c}$ ). By carefully controlling the volumetric inlet flow rate $(r)$ for stable base line, modulated signals of the injected permeate molecules are obtained at a designed time interval. A sharp and discrete outlet signal is verified for a detectable concentration of permeate at a given experimental condition. As long as the flow rate $(r)$ is fixed, normalized frequency of outlet to inlet $\left(f_{\text {out }} / f_{\text {in }}\right)$ maintains unity (Figure $2 \mathrm{c}$ and $2 \mathrm{~d}$ ). However, the $\mathrm{R}_{\mathrm{t}}$ decreases linearly as $r$ increases (Figure $2 \mathrm{~d}$ ). $\mathrm{R}_{\mathrm{t}}$ exhibits a typical linear relation according to the van Deemter equation as follows: $L \sim D_{1}+D_{2} / r+D_{3} r$. In this equation, $L$ is the permeate path length, $r$ is the flow rate, $D_{1}$ is the multiple diffusion parameter, $D_{2}$ is the longitudinal diffusion and $D_{3}$ is the mass transport parameters ${ }^{43}$. When the mass transfer effect is dominant, which corresponds to the system designed in this study, $\mathrm{R}_{\mathrm{t}}$ is inversely proportional to the $r$ in a linear relation. When the flow rate is controlled from 0.01 to $0.1 \mathrm{~cm}^{3} / \mathrm{min}, \mathrm{R}_{\mathrm{t}}$ changes from $16 \mathrm{~min}$ to $4 \mathrm{~min}$ (Figure $2 \mathrm{~d}$ ). When the total volume of the column as of $0.08 \mathrm{~cm}^{3}$, the efficiency factor $(H)$ passing through the designed nanocomposite is close to 2 based on the following relation: $H=L$ $\times\left[\left(\mathrm{R}_{\mathrm{t}(\text { permeate) }}-\mathrm{R}_{\mathrm{t}(\text { solvent })}\right) / \mathrm{R}_{\mathrm{t}(\text { solvent })}\right]$.

Flow-controlled mass transport. Representative synchronized frequency patterns are displayed where the two inlet $\left(f_{\text {in }}\right)$ and outlet $\left(f_{\text {out }}\right)$ frequencies are highly synchronized (Figure $2 \mathrm{~B}$ and 2c). At different $r$ values $\left(0.01\right.$ to $\left.0.1 \mathrm{~cm}^{3} / \mathrm{min}\right)$, the normalized frequency $\left(f_{\text {out }} / f_{\text {in }}\right)$ pattern is constant $\left(f_{\text {out }} / f_{\text {in }}=1\right)$ as long as the acceleration rate is zero $\left(a=\mathrm{d} r_{\text {in }} / \mathrm{dt}=0\right)$. However, when $r_{\text {in }}$ is continuously changed at a fixed acceleration $\left(a=\mathrm{d} r_{\text {in }} / \mathrm{dt}\right), f_{\text {out }} / f_{\text {in }}$ varies systematically (Figure $3 \mathrm{a}$ ). Under the given condition, the variation of $f_{\text {out }} / f_{\text {in }}$ exhibits linear relation according to $a$ (Figure $3 \mathrm{~b})$. At a given pass length condition $(L)$, this linear relation with $a$ directly indicates the linear proportionality to the kinetic energy. Therefore, the designed mass (rhodamine 6G) flow behaves as a conventional mass for which the general physical laws are applied: $E($ energy $) \sim F($ force $) \times L$ (length) $\sim m$ (mass) $\times a$ (acceleration $) \times L$. As $r_{\text {in }}$ continuously increases or decreases at a constant permeate injection frequency $\left(f_{\text {in }}=4 \mathrm{~min}^{-1}\right), f_{\text {out }}$ increases or decreases accordingly. The results for two representative cases are displayed in Figure 3a. As $r$ changes with different $a= \pm 0.005 \mathrm{~cm}^{3} / \mathrm{min}^{2}$ and $a$ $= \pm 0.01 \mathrm{~cm}^{3} / \mathrm{min}^{2}$, the variations in $f_{\text {out }}$ are displayed at the middle and the right graphs in Figure 3a. Under no acceleration $(a=0)$, the inlet frequency $\left(f_{\text {in }}\right)$ and the first outlet frequency $\left(f_{\text {out }}\right)$ has no difference $\left(f_{\text {out }} / f_{\text {in }}=1\right)$. The normalized frequency $\left(f_{\text {out }} / f_{\text {in }}\right)$ exhibit linear proportionality for continuous change of $a$. This finding is attributed to to the mass-energy relation where the energy $(E)$ is proportional to volumetric acceleration $(a): E \sim a$. Therefore, the volumetric acceleration/deceleration, rather than the absolute flow rate, has a significant influence on the permeate output. Even for densely packed viscous soft condensed matter media designed in this 

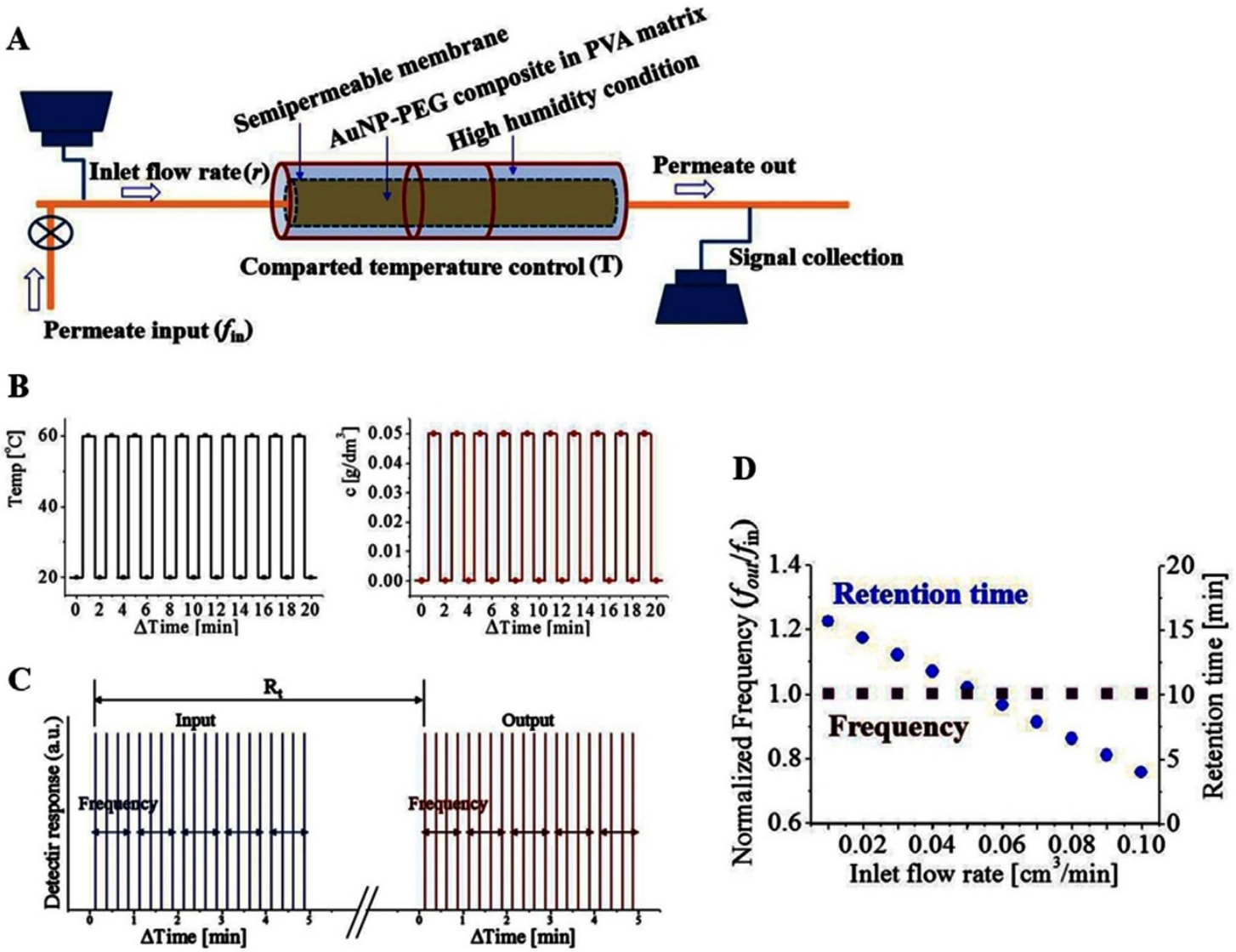

Figure $2 \mid$ (a) Experimental set-up for mass-transport. Permeate molecules of periodic injection $\left(f_{\text {in }}\right)$ are loaded with a controlled flow rate $(r)$. (b) Temperature control (left) and molecular signal detection (right) are highly synchronized, indicating stably optimized system for mass transport investigation. (c) Retention time $\left(\mathrm{R}_{\mathrm{t}}\right)$ during which the permeate molecules are stay in the cell before they are detected at the outlet. At a fixed $r$, the input frequency $\left(f_{\text {in }}\right)$ and output frequency $\left(f_{\text {out }}\right)$ are kept same. $(\mathrm{d}) \mathrm{R}_{\mathrm{t}}$ decreases linearly, whereas normalized frequency $\left(f_{\text {out }} / f_{\text {in }}\right)$ maintains unity according to $r$.

study, the energy (E) relation is satisfied as the permeates freely pass through

$$
\left(f_{\text {out }} / f_{\text {in }}\right) \sim E=m a L
$$

The passing molecules are accelerated following the conventional mass-energy relation applied in typical particle-like physical behaviors.

Mass transport by contractile actions of one-valve system. With a fixed $f_{\text {in }}=4\left(\mathrm{~min}^{-1}\right)$ and $r=0.01 \mathrm{~cm}^{3} / \mathrm{min}$, temperature at the middle of the designed nanocomposite pellet is controlled (Figure 4a). The off position in the valve function is provided by switching the temperature from $60^{\circ} \mathrm{C}$ to $20^{\circ} \mathrm{C}$. The cooling process is only applied to the middle section on $2 \mathrm{~cm}$ length region (out of $10 \mathrm{~cm}$ total tube length) to induce temperature gradient. The frequency maintains a regular pattern $\left(4 \mathrm{~min}^{-1}\right)$ before changing to the offstate. However, during the responding time $\left(t_{\text {respond }}\right)$, permeate movement is suddenly delayed by the contractile actions in the offstate. This phenomenon is caused by the swelling procedure of the nanocomposite, which increases the back pressure caused by sudden water absorption. After the abrupt stop of permeate movement, the decreased water flow induces an incremental decrease in $f_{\text {out }}$, forming a tapering zone $\left(t_{\text {taper }}\right)$. Once most of the permeates exit the off-zone, the permeate elution is completely stopped until the designed $t_{\text {off }}$ ends. When the on-position state and flow are both regained after $t_{\text {off, }}$, the permeates are detected in a high frequency $\left(f_{\text {recover }}\right)$ because of accumulative congestion during the period of $t_{\text {off }}$.
The frequency stabilizes during the recovering time $\left(t_{\text {recover }}\right)$ to regain original frequency $\left(f_{\text {in }}=f i\right)$, where fi is the $i$ th frequency after $f_{\text {recover }}$.

With varying duration of $\mathrm{t}_{\mathrm{off}}$, the resulting $f_{\text {out }}$ of the designed systems is observed accordingly (Figure $4 \mathrm{~b}$ ). $t_{\text {off }}$ is controlled from $15 \mathrm{~s}$ to 3 minutes in the middle of the stable flow at a fixed $r$. $t_{\text {respond }}[\mathrm{I}]$ and $t_{\text {taper }}[\mathrm{II}]$ are independent of $t_{\text {off }}$, which reflects the characteristic property of the nanocomposite itself. Based on the increase in the $t_{\text {off }}$, the first maximum recovering frequency $\left(f_{\text {recover }}\right)$ right after the $t_{\text {off }}$ is normalized by the inlet frequency $\left(f_{\text {recover }} / f_{\text {in }}\right)[\mathrm{III}]$. This normalized value is saturated from a specific point $\left(\mathrm{d}\left(f_{\text {recover }} / f_{\text {in }}\right) / \mathrm{dt}=0\right)$ at which the optimum off-time $\left(t_{\text {opt }}\right)$ is determined. This finding implies a limit to increase the mass transport rate continuously by increasing the $t_{\text {off. Thus an }}$ optimized contractile action exist. To find the critical point from which the increase of the mass transport is limited (or saturated), variations in $f_{\text {recover }} / f_{\text {in }}$ is investigated with varying parameters (Figure 5c). With decreasing the $r$, the time to reach the critical point takes longer. Before reaching the critical point, the $f_{\text {recover }} /$ $f_{\text {in }}$ relation follows an approximate liner relation with a definable slope $\alpha$ as a function of $r$ : $\left(f_{\text {recover }} / f_{\text {in }}\right)=\alpha(r) \mathrm{t}_{\text {off }}+\phi$, where $\phi$ is an intercept (Table S1). However, high $f_{\text {in }}$ only increases the magnitude of the $f_{\text {recover }} / f_{\text {in }}$ whereas the critical point remains unchanged. Therefore, to determine the optimized $t_{\text {off }}, r_{\text {in }}$ is exclusively decisive, and $f_{\text {in }}$ contributes to the magnitude of the $f_{\text {recover }} / f_{\text {in }}$. Therefore, the mass transport is controlled by the energy impartment conveyed by the flow acceleration. Since $f_{\text {recover }} / f_{\text {in }}$ is linearly proportional to $r$, the following relation is 

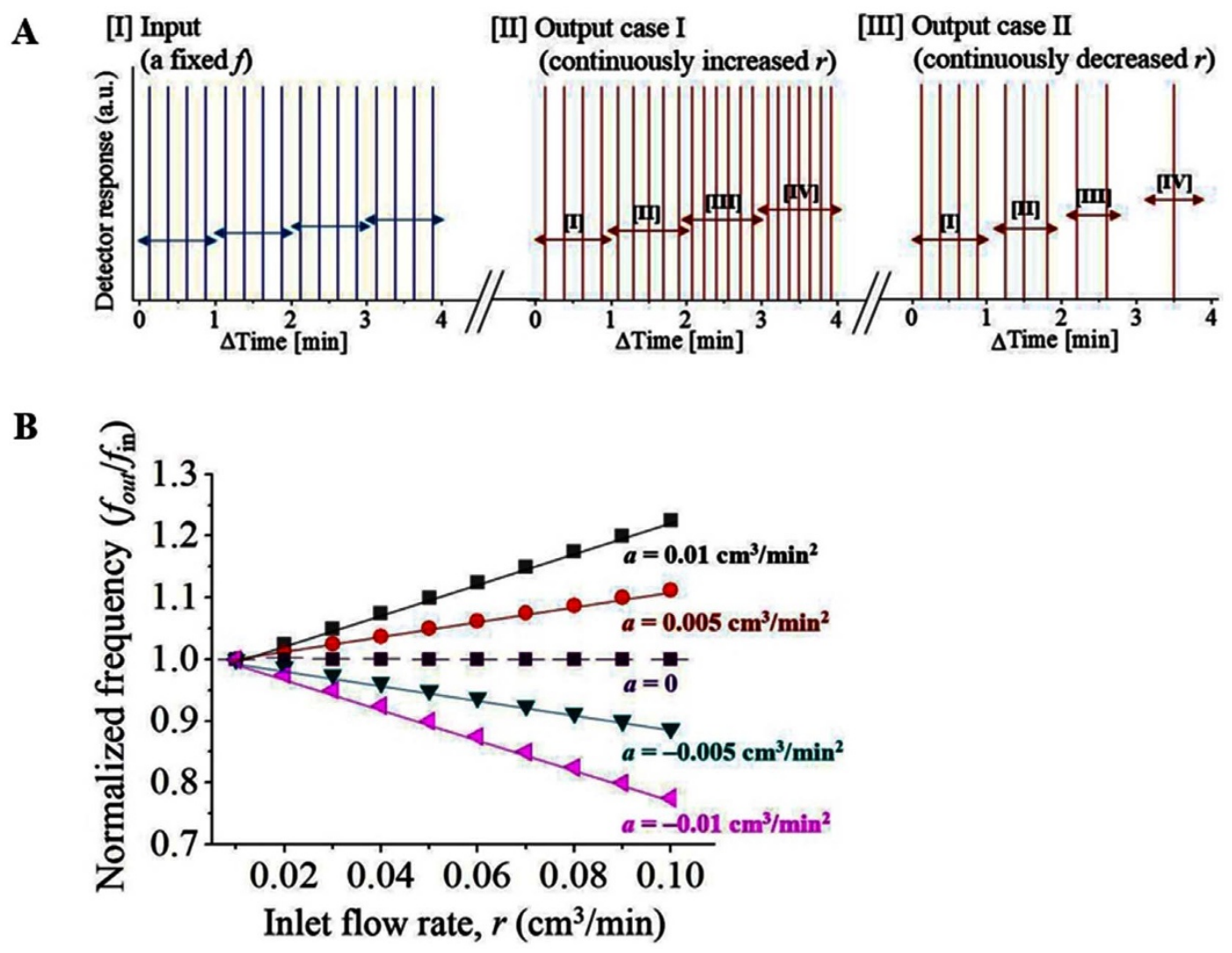

Figure $3 \mid$ (a) Representative result of $f$ change according to the designed $r$ ( $a$, acceleration). [I] Input at a fixed $f$ and designed $r$. [II] Output case I: $f_{\text {out }}$ increases with continuously increasing $r$. [III] Output case II: $f_{\text {out }}$ decreases with continuously decreasing $r$. (b) Graphical summary of the results obtained in (a). Without acceleration $(a=0), f_{\text {out }} / f_{\text {in }}$ maintains unity according to $r . f_{\text {out }} / f_{\text {in }}$ linearly increases with positive $a$, but linearly decreases with negative $a$. These results exhibit typical particle-like behavior of permeate molecules under acceleration caused by increased $r$. Considering the fixed pathlength of the system, the result reflects the linear mass-energy relation.

reasonably satisfied:

$$
\left(f_{\text {recover }} / f_{\text {in }}\right) \sim E^{1 / 2}
$$

Compared with Eq. (1), which is applicable to stable flows linearly proportional to $E$ without contractile action, the normalized maximum outlet frequency $\left(f_{\text {recover }} / f_{\text {in }}\right)$ is proportional to the half power of $E$ when a contractile action is applied.

Figure $4 \mathrm{~d}$ shows the variations of the time duration elapsed to recover the original frequency $\left(\mathrm{t}_{\text {recover }}\right)$ plotted by $\mathrm{t}_{\text {off. }}$. The time duration is classified into two regions, namely $t_{\text {off }}$ and $t_{\text {opt }}$ :

$$
\begin{gathered}
\mathrm{K}(r) \mathrm{t}_{\text {off }}+1 / f_{\text {in }} \quad \text { at } \mathrm{t}_{\text {off }} \leq \mathrm{t}_{\text {opt }} \\
\mathrm{K}(r)\left[\mathrm{t}_{\text {off }}+\left(\mathrm{t}_{\text {off }}-\mathrm{t}_{\text {opt }}\right)\right]+1 / f_{\text {in }} \quad \text { at } \mathrm{t}_{\text {off }}>\mathrm{t}_{\text {opt }}
\end{gathered}
$$

In Eq. (3), $t_{\text {recover }}$ is linearly proportional to $t_{\text {off. }}$ In Eq. (4), the difference between the actually performed off-time and the optimum off-time $\left(t_{\text {off }}-t_{\text {opt }}\right)$ is additionally inlcuded. $t_{\text {optinum }}$ is the suggested time for which the mass transport rate is maximized, and an extended time period does not increase the mass transport rate. Therefore, $t_{\text {recover }}$ is necessary to deliver the overloaded masses. The obtained experimental results are fitted by the linear relation of Eq. (3). In additin, the proprotional factor $\mathrm{K}(r)$ is a fucntion of $\boldsymbol{r}$ satisfying a liner relation with $r$. An intercept value of $1 / f_{\text {in }}$ explains that the $\mathrm{t}_{\text {recover }}$ is inversely proportional to the $f_{\text {in }}$ in all the cases in this study. The concept of the optimized periodicity thus summarized in Figure 4e. One period is composed of $t_{\text {optinum }}$ and $t_{\text {recover }}$ which are the functions of the $\mathrm{t}_{\text {off }}, f_{\text {recover }}$ and $f_{\text {in }}$.
Mass transport by contractile actions in a series of multi-valve system. Mass transport is controlled by consecutive repeated contractile actions (Figure 5a). With a series of the 10 compartments, each section is sequentially heated to generate a partial blockage during the interval time ( $\left.t_{\text {interval }}\right)$ from $t_{1}$ to $t_{10}$ under the conditions of $t_{1}=t_{2}=t_{3}=\ldots=t_{10}$. When the contractile actions are performed in a series, a pushing effect on a specific point occurs, illustrated as arrows on the left-side. Heating is applied on each compartmental section of the temperaturecontrolled tube on the right in a series, so consecutively moving contractile actions can be generated. The stability of the system is verified by checking whether the normalized frequency $\left(f_{\text {out }} / f_{\text {in }}\right)$ of the system maintains unity without temperature modulation (Figure $5 b)$. When the total elapsed time $t_{\text {interval }}=\Sigma t_{i}(i=1,2,3, \ldots 10)$ is determined, then the temperature frequency is defined as $f_{\text {temp }}=1 /$ $t_{\text {interval. }}$. When a partial contractile action is applied in a series (Figure $5 \mathrm{c}$ ), the mass transport can be either promoted or prohibited. With the increase in inlet flow rate $(r), f_{\text {out }} / f_{\text {in }}$ value increases until a specific frequency (i.e., $f_{\text {temp }}=0.3$ ) and then significantly drops down. In addition, $f_{\text {out }} / f_{\text {in }}$ is proportional to the square of the $r$ as curve-fitted in the graph. The experimental conditions and the fitting results are available in Supporting Information (Table S2). This result indicates that mass transport is accelerated linearly proportional to the energy as follows:

$$
\left(f_{\text {out }} / f_{\text {in }}\right) \sim r^{2} \sim E
$$

Therefore, high synchronization of the contractile actions performed in a series is necessary to match with the fluid flow rate. 
$\mathbf{A}$
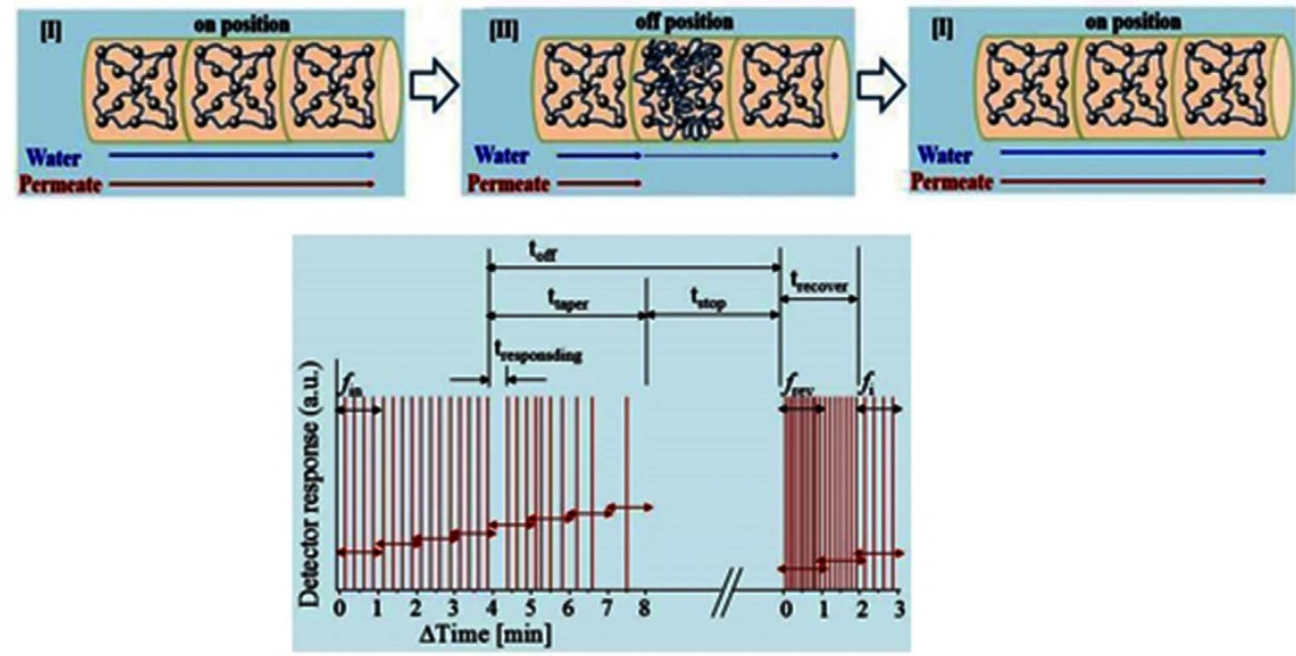

B

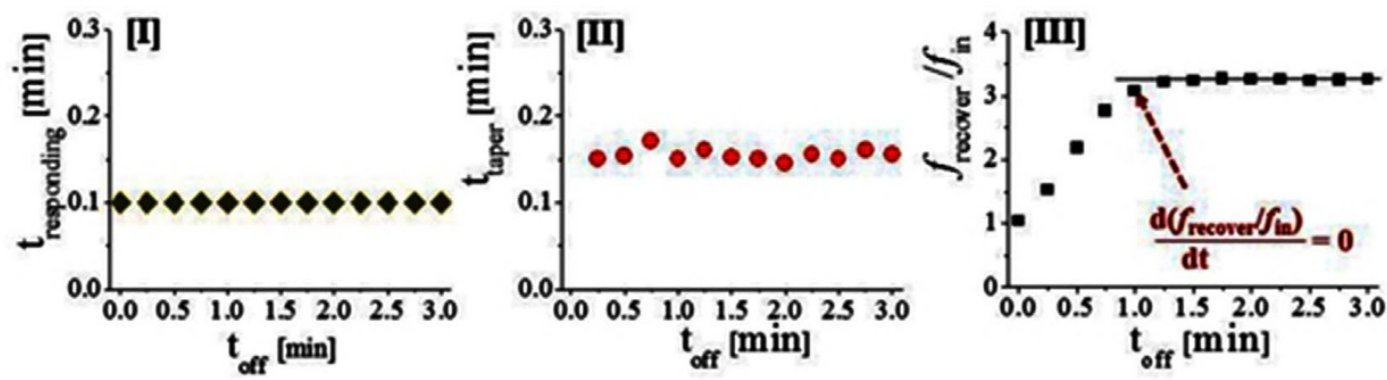

C
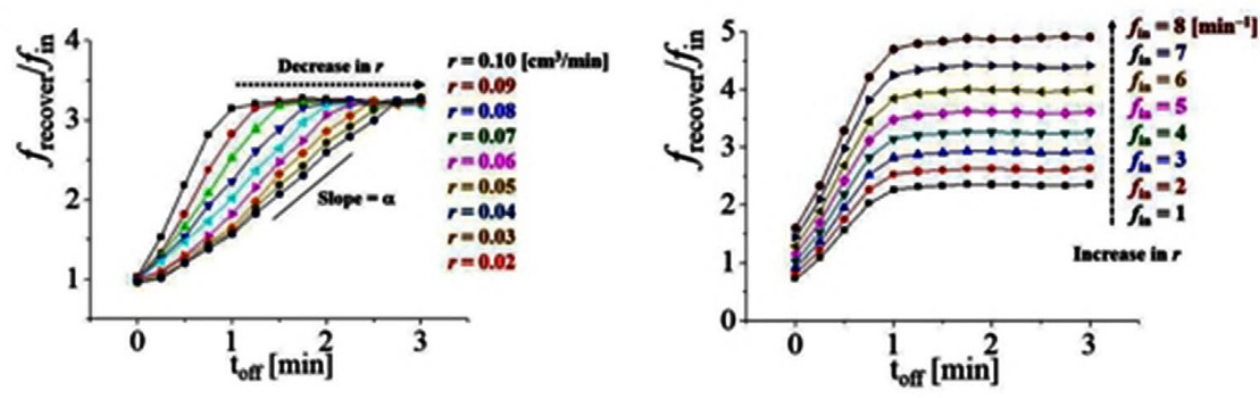

D

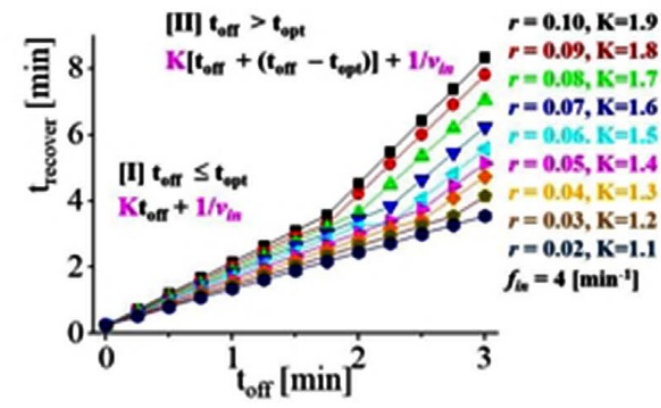

$\mathbf{E}$

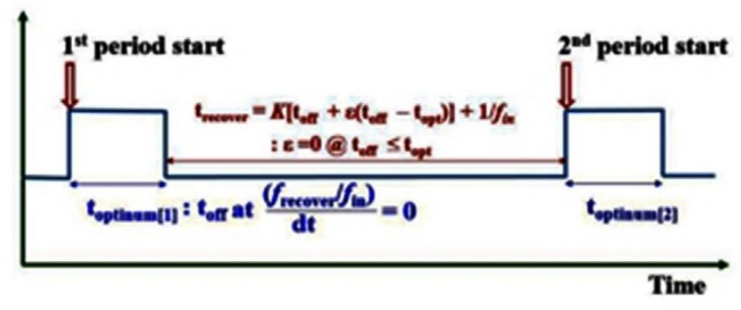

Figure $4 \mid$ (a) Design of the contractile action on flow. By repeating on-off-on position, one valve function is designed (upper). Representative data of the one-valve system. During the designed off-time $\left(t_{\text {off }}\right)$, the inlet flow stops. During the taper time $\left(t_{\text {taper }}\right)$, detection of permeate molecules slows followed by complete stop-time $\left(t_{\text {stop }}\right)$. Right after the $t_{\text {off }}$ starts, there is a responding time $\left(t_{\text {responding }}\right)$ at which the flow stops suddenly and generates back pressure inside the cell. According to the inlet frequency $\left(f_{\text {in }}\right)$, the outlet frequency is modulated. Right after the $t_{\text {off }}$, the first maximum outlet frequency is determined as recover frequency $\left(f_{\text {recover }}\right)$ after that $i^{\text {th }}$ frequency is determined in a sequence. (b) [I] $t_{\text {off }}$ vs. $t_{\text {responding }}[\mathrm{II}] \mathrm{t}_{\text {off }} \mathrm{vs.} \mathrm{t}_{\text {taper }}$. $t_{\text {responding }}$ and $t_{\text {taper }}$ are independent of $t_{\text {off }}$, reflecting system-specific features. [III] $t_{\text {off }}$ vs. maximum normalized frequency $\left(f_{\text {recover }} / f_{\text {in }}\right)$. The frequency increase is saturated at a critical point, so the optimum time $\left(\mathrm{t}_{\text {optinum }}\right)$ is determined. (c) $\mathrm{t}_{\text {off }} \mathrm{vs}$. $f_{\text {recover }} / f_{\text {in }}$ with changing $r$ (left $)$ and $f_{\text {in }}($ right $)$. The critical point move to higher $\mathrm{t}_{\text {off }}$ by decreasing $r$, whereas a higher $f_{\text {in }}$ contributes to the increase in the magnitude of $f_{\text {recover }} / f_{\text {in }}$. (d) Fitting results of the $t_{\text {recover }}$ vs. $\mathrm{t}_{\text {off }}$ in the two regions: [I] in the range of $\mathrm{t}_{\text {off }} \leq \mathrm{t}_{\text {opt }}, \mathrm{K}(r) \mathrm{t}_{\text {off }}+1 / f_{\text {in }}$ relation is satisfied. [II] in the range of $\mathrm{t}_{\text {off }}>\mathrm{t}_{\text {opt }}, \mathrm{K}(r)\left[\mathrm{t}_{\text {off }}+\left(\mathrm{t}_{\text {off }}-\mathrm{t}_{\text {opt }}\right)\right]+1 / f_{\text {in }}$ relation is applied. The proportional factor $\mathrm{K}$ is also a function of $r$. (e) Summary of one periodicity composed of $\mathrm{t}_{\text {off }}$ and $t_{\text {recover }}$ as a function of $t_{\text {off }}$, $\mathrm{t}_{\text {optinum }}, f_{\text {recover }}$ and $f_{\text {in }}$. 
$\mathbf{A}$

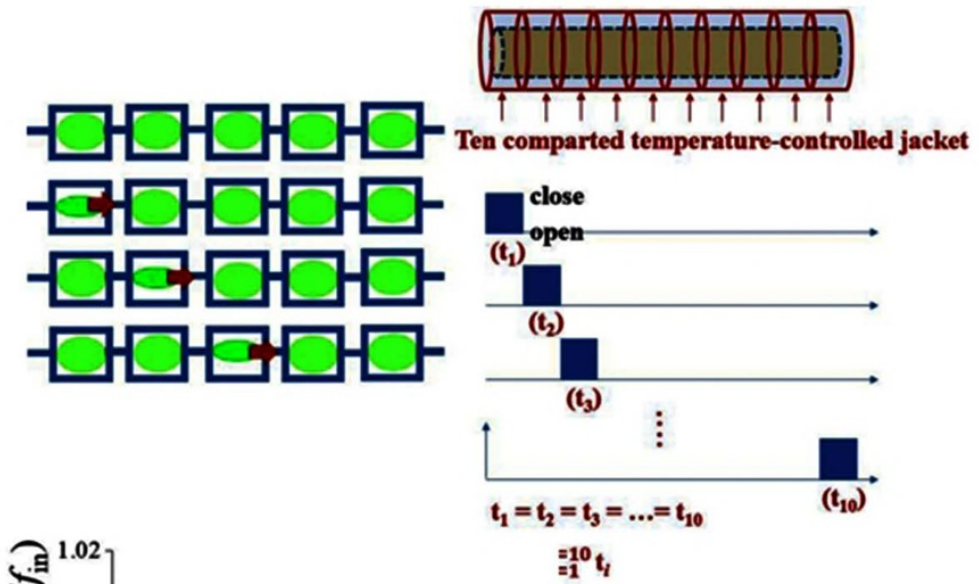

B

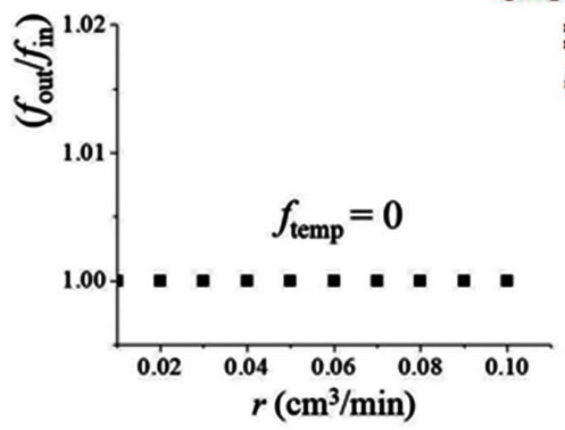

$\mathbf{1 / t}$

C
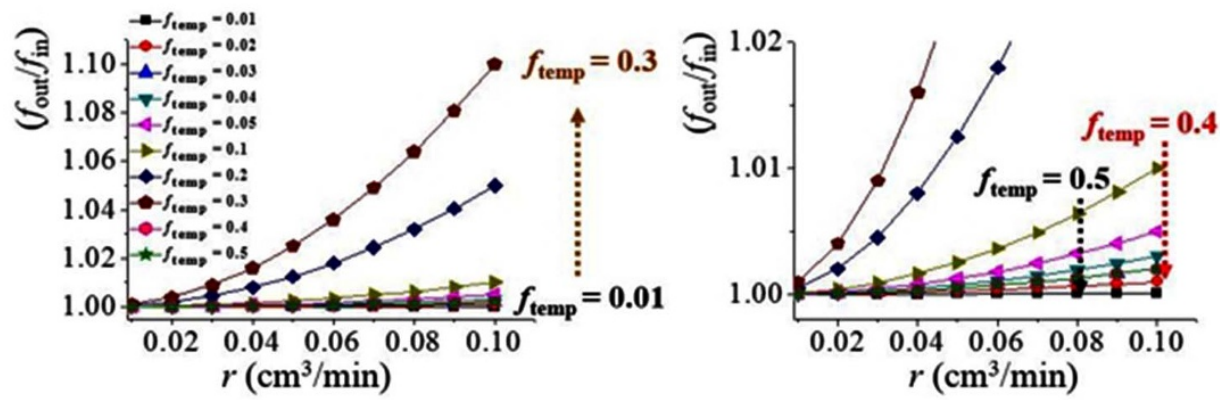

Figure $5 \mid$ (a) Schematic illustration of the multi-valve system of sequential contractile actions. The cell is divided into 10 units of individual temperature control. The time for each unit is designed to be same as $t_{1}=t_{2}=t_{3} \ldots \ldots=t_{10}$. The temperature frequency $\left(f_{\text {temp }}\right)$ is determined as inverse proportionality of the total $t=\sum_{i=1}^{i=10} t_{i}$ time taking for one cycle. (b) Without $f_{\text {temp }}$ introduction, the system exhibits stable $\left(f_{\text {out }} / f_{\text {in }}\right)=1$. (c) With increased $f_{\text {temp }}$ from 0 to $0.3\left(\mathrm{~min}^{-1}\right)$, the $f_{\text {out }} / f_{\text {in }}$ increases proportionally to $r^{2}$. However, $f_{\text {out }} / f_{\text {in }}$ decreases significantly from $f_{\text {temp }}=0.4 \mathrm{~min}{ }^{-1}$. Therefore, an optimum $f_{\text {temp }}$ is necessary to maximize the $f_{\text {out }} / f_{\text {in }}$.

\section{Discussion}

Mass transport has been investigated typically based on diffusion process for which Fick's law is applied ${ }^{21}$. Transport through smallscale pores, such as reverse osmosis membranes, has been described by the solution-diffusion model ${ }^{22}$ and other modelling techniques ${ }^{23}$. In addition, small ions and large-scale colloidal particles in size ranges of 1 to $100 \mathrm{~nm}$ have been analyzed in a single theoretical framework ${ }^{24}$. Although the discrete nature of individual nanoscale molecules is evident, the main transport phenomena in nanofluidic systems are explained based on the continuum and mean-field approaches. Unlike these diffusion-dominant passive concepts, a new molecular transport is recapitulated in this study based on the acceleration by repeated contractile action in fluid flow. Depending on the contractile styles (one-valve and continuous multi-valve systems), the amount of energy imparted by the contractile actions is different. Under continuously increased flow rate (with a fixed acceleration) or sequential contractile multi-valve motion, the mass transport efficiency expressed by normalized frequency $\left(f_{\text {out }} / f_{\text {in }}\right)$ is linearly proportional to the energy: $\left(f_{\text {out }} / f_{\text {in }}\right) \sim E$. Regarding the contractile action using a onevalve system, the relation $\left(f_{\text {out }} / f_{\text {in }}\right) \sim E^{1 / 2}$ is satisfied.
Contractile energy is employed in many natural and artificial systems. Blood flow activated by heart beats and undulatory actions of intestines are representative contractile-promoted actions occurring in biological systems. This repeated contractile phenomena actually occur in the human body. The natural defensive mechanism of the human body such as response to the hypotension hemorrhage and blood vessel occlusion, might be explained based on the physical phenomena occurring in the optimal model. In practical artificial systems, intermittent flow controlled systems are generally relevant to this concept. We can successfully reenact the important dynamic behaviors occurring natural systems by effectively employing stimuli-responsive soft condensed materials ${ }^{25}$. This study would contribute to the basic and general understanding on the optimal mass transport in the dynamics-promoted flow systems.

\section{Methods}

Permeate molecule transport through AuNP-PEG nanocomposites. The volume and height of the AuNP-PEG nanocomposite embedded in the PVA matrix ( $3 \mathrm{wt} \%$, $\mathrm{Mw}=72,000$; Merck) is designed as a pellet (diameter $=0.1 \mathrm{~cm}$; length $=10 \mathrm{~cm}$ ). The nanocomposite pellets are loaded in a thin-walled $(0.01 \mathrm{~mm})$ glass tube (Hampton Research, CA, USA) in which the humidity and temperature are carefully 
controlled. Water flow rate $(r)$ and mass transport frequency $(f)$ are controlled through the designed nanocomposites. Rhodamine $6 \mathrm{G}$ is an ionic molecule sensitive to laser-based detection. It exhibits a dark orange color in water, so it is used as an effective tracer in water flow. With high water solubility $(400 \mathrm{~g} / \mathrm{L})$, rhodamine $6 \mathrm{G}$ is directly dissolved in water and used as a saturated solution. Concentration-controlled permeate solutions are injected into the inlet line at the designed time interval (MicroLiter OEM Syringe Pump Modules, Harvard Apparatus, MA, USA). A series of eluted permeates are measured and recorded using a spectrophotometer $(2489 \mathrm{UV} /$ visible detector, Waters, MA, USA). Empower 3 Chromatography Data Software is employed for data analysis. Spectra are obtained at a scan rate of $100 \mathrm{~nm} / \mathrm{s}$, and intensities are collected at an interval of $0.5 \mathrm{~nm}$. For long-term and quick scanning, the wavelength range is narrowly controlled for rhodamine 6G spectra at an excitation wavelength of $485 \mathrm{~nm}$. Scanning is performed every $5 \mathrm{~s}$ so that the possible maximum frequency designed in this study is evaluated as $12 \mathrm{~min}^{-1}$. All the spectra are obtained at $20^{\circ} \mathrm{C}$. To precisely control the local temperature of the designed nanocomposites, a multi-point temperature controller (MPC, Briskheat Corp., $\mathrm{OH}$, USA) with resistance heating coils made of coiled chromel wire (MOR Electric Heating Assoc. Inc., MI, USA) is employed.

Small-angle X-ray scattering (SAXS). SAXS. Synchrotron SAXS measurements are obtained at the $4 \mathrm{C}$ beamline at PAL equipped with a position-sensitive twodimensional (2D) detector. Two energy settings are employed for wavelength modulation: $10\left(0.0675 \mathrm{~nm}^{-1}\right)$ and $18 \mathrm{keV}\left(0.1217 \mathrm{~nm}^{-1}\right)$. Samples of $1 \mathrm{~mm}$-thick are used by stacking five $200 \mu \mathrm{m}$-thick Si wafer with SiN3 sample window (window thickness is $1 \mu \mathrm{m}$ so that it hardly contributes to the sample thickness). The sampleto-detector distance (SDD) of $4 \mathrm{~m}$ covers the $\mathrm{q}$ range of $0.0679 \mathrm{~nm}^{-1}<\mathrm{q}<$ $1.64094 \mathrm{~nm}^{-1}$, where $q=(4 \pi / \lambda) \sin (\theta / 2)$ is the magnitude of the scattering vector and $\theta$ is the scattering angle. The $q$ range is calibrated using polystyrene-blockpoly(ethylene-ran-butylene)-block-polystyrene (SEBS) $\left(q=0.19165 \mathrm{~nm}^{-1}\right)$. On the other hand, the $1 \mathrm{~m} \mathrm{SDD}$ covers the $q$ range of $0.346 \mathrm{~nm}^{-1}<q<7.68039 \mathrm{~nm}^{-1}$. The $q$ range is calibrated using silver behenate $\left(q=1.052 \mathrm{~nm}^{-1}\right)$. During the measurements, the temperature is precisely controlled at an isothermal condition of $20^{\circ} \mathrm{C}$ if no heating plan is involved. To increase the sample temperature to $40^{\circ} \mathrm{C}$ and $60^{\circ} \mathrm{C}$, programmed heating coil is employed to connect to the copper-based sample holder body. A W/B4C double multilayer monochromator is installed to deliver monochromatic X-rays with a wavelength of $6.75 \mathrm{~nm}(18360 \mathrm{keV})$ and spread of $\Delta \lambda /$ $\lambda=0.01$. The $2 \mathrm{D}$ scattered X-rays are recorded by a CCD camera (Mar CCD, Mar USA, Inc., CCD165). The collected SAXS data are corrected by subtracting the background and empty cell scattering.

Synchrotron X-ray nanoscopy $(\mathrm{XN})^{26}$. Experiments are carried out at the $7 \mathrm{C}$ beamline of PAL. The X-ray source of $10^{11}$ photons $/ \mu \mathrm{m}^{2} / \mathrm{sec}$ consists of undulator with $20 \mathrm{~mm}$ period and 70 poles. The beam size is about $100 \mu \mathrm{m} \times 100 \mu \mathrm{m}$ at $7 \mathrm{keV}$. The $\mathrm{X}$-ray source is radiated from a $3 \mathrm{GeV}$ bending magnet and then monochromatized using a Ge(111) DCM. To achieve focused images, monochromatic X-ray beam of nominally selected $7 \mathrm{keV}$ is focused on the sample using a condenser zone-plate (CZP, $1 \mathrm{~mm}$ dia. Beryllium refractive compound lenses) with innermost and outermost diameters of $4 \mathrm{~nm}$ and $100 \mathrm{~nm}$, respectively. The primary X-ray image is magnified 50 times with an objective zone plate lens (140 $\mu \mathrm{m}$ innermost and $50 \mathrm{~nm}$ outermost diameter, W). It is then converted into a visible image on a thin scintillator crystal (Tb:LSO, $20 \mu \mathrm{m}$ thickness). The visible image is further magnified $\times 20$, using an optical microscope. This provides a total $\times 1000$ magnification of image on a cooled CCD camera (Princeton Instrument VersArray $1300 \mathrm{~B}$ cooled CCD) of $1340 \times 1300$ pixels, which corresponds to an equivalent $\mathrm{FOV}$ of $21 \times 21 \mu \mathrm{m}^{2}$.

Synchrotron X-ray microscopy (XM). Synchrotron X-ray images are captured at 6D beamline of PAL. The X-ray source is a bending magnet with critical energy of $8.7 \mathrm{keV}$ at $3 \mathrm{GeV}$ electron energy operation. The white beam is attenuated by polished beryllium (Be) of $0.5 \mathrm{~mm}$ thickness and polished Si wafer of $1 \mathrm{~mm}$ thickness. The primary X-ray image is converted into a visible image on the thin scintillator crystal $\mathrm{CdWO}_{4}$ of $100 \mu \mathrm{m}$ thickness. X-ray images are captured using a CCD camera (Vieworks, VH-2MC). With a $10 \times$ objective lens attached in front of the camera generates the field-of-view of $1.2 \mathrm{~mm} \times 0.9 \mathrm{~mm}$ in physical dimension. The size of each pixel is about $0.74 \mu \mathrm{m}$.

1. Kasahara, J. Tides, earthquakes, and volcanoes. Science 297, 348-349 (2002).

2. Mendiguren, J. A. High-resolution spectroscopy of the Earth's free oscillations, Knowing the earthquake source mechanism. Science 12, 179-180 (1973).

3. O'Neil, J. S. \& Reddy, A. B. Circadian clocks in human red blood cells. Nature 469 498-503 (2011).

4. Kondratova, A. A. \& Kondratov, R. V. The circadian clock and pathology of the ageing brain. Nat. Rev. Neurosci. 13, 325-335 (2012).

5. Streeter, V. L., Keitzer, W. F. \& Bohr, D. F. Pulsatile pressure and flow through distensible vessels. Circulation Research 13, 3-20 (1963).
6. Capadona, J. R., Shanmuganathan, K., Tyler, D. J., Rowan, S. J. \& Weder, C. Stimuli-responsive polymer nanocomposites inspired by the sea cucumber dermis. Science 7, 1370-1374 (2008).

7. Bhattacharyya, D., Schafer, T., Wickramasinghe, S. R. \& Daunert, S. Responsive membranes and materials. Wiley, New York (2013).

8. Ahn, S., Ahn, S. W. \& Song, S. C. Thermothickening modification of the poly(ethylene glycol) and amino acid ester grafted polyphosphazenes by monomethyl end-capped poly(ethylene glycol) addition. Colloid Surf. APhysicochem. Eng. Asp. 333, 82-90 (2009).

9. Ahn, S., Ahn, S. W. \& Song, S. C. Thermosensitive amphiphilic polyphosphazenes and their interaction with ionic surfactants. Colloid Surf. A-Physicochem. Eng. Asp. 330,184-192 (2008).

10. Skorb, E. V., Sviridov, D. V., Möhwald H. \& Shchukin, D. G. Light responsive protective coatings. Chem. Commun. 40, 6041-6043 (2009).

11. Frederickson, R. Test run for kidney replacement. Nat. Biotechnol. 17, 413 (1999)

12. Woodle, M. C., Engbers, C. M. \& Zalipsky, S. New amphipatic polymer-lipid conjugates forming long-circulating reticuloendothelial system-evading liposomes. Bioconjugate Chem. 5, 493-496 (1994).

13. Ahn, S., Jung, S. Y., Lee, J. P., Kim, H. K. \& Lee, S. J. Gold nanoparticle flow sensors designed for dynamic X-ray imaging in biofluids. ACS Nano 4, 3753-3762 (2010).

14. Mirkin, C. A., Letsinger, R. L., Mucic, R. C. \& Storhoff, J. J. A DNA-based method for rationally assembling nanoparticles into macroscopic materials. Nature 382, 607-609 (1996).

15. Elghanian, R., Storhoff, J. J., Mucic, R. C., Letsinger, R. L. \& Mirkin, C. A. Selective colorimetric detection of polynucleotides based on the distance-dependent optical properties of gold nanoparticles. Science 277, 1078-1081 (1997).

16. Ahn, S., Ahn, S. W. \& Song, S. C. Polymer structure-dependent ion interaction studied by amphiphilic nonionic poly(organophosphazenes). J. Polym. Sci. Pt. B Polym. Phys. 46, 2022-2034 (2008).

17. Ahn, S., Monge, E. C. \& Song, S. C. Ion and pH effect on the lower critical solution temperature phase behavior in neutral and acidic poly(organophosphazene) counterparts. Langmuir 25, 2407-2418 (2009).

18. Csetneki, I., Filipcsei, G. \& Zrinyi, M. Smart nanocomposite polymer membrane with on/off switching control. Macromolecules 39, 1939-1942 (2006).

19. Schäfer, F. P. (Ed.), Dye Lasers, 3rd Ed. Springer-Verlag, Berlin( 1990).

20. Dong, D. C. \& Winnik, M. A. The Py scale of solvent polarities. Can. J. Chem. 62, 2560-2565 (1984).

21. Fick, A. On Liquid Diffusion. Phil. Mag. J. Sci. 10, 30-39 (1855).

22. Wijmans, J. G. \& Baker, R. W. The solution-diffusion model - a review. J. Membr. Sci. 107, 1-21 (1995).

23. Gad-el-Hak, M. The fluid mechanics of microdevices - The Freeman scholar lecture. J. Fluid Eng.-T. ASME 121, 5-33 (1999).

24. Sparreboom1, W., van den Berg, A. \& Eijkel, J. C. T. Principles and applications of nanofluidic transport. Nat. Nanotechnol. 4, 713-720 (2009).

25. Ahn, S. \& Lee, S. J. Nanoparticle role on the repeatability of stimuli-responsive nanocomposites. Scientific Reports (2014) in press.

26. Lim, J., Kim, H. \& Park, S. Y. Hard X-ray nanotomography beamline 7C XNI at PLS-II. J. Synchrot. Radiat. 21, 827-831 (2014).

\section{Acknowledgments}

This work was supported by the National Research Foundation of Korea grant funded by the Korea government (MSIP) (No. 2008-0061991). The authors are grateful for the valuable help in the synchrotron X-ray experiments performed at the 6D XMI, 7C X-ray XNI and 4C SAXS of the Pohang Accelerator Laboratory (Pohang, Korea).

\section{Author contributions}

S.A. and S.J.L. developed the concepts. S.A. designed and perform the experiments. S.A. analyzed the results and wrote the paper. All the authors confirm the final version of the manuscript.

\section{Additional information}

Supplementary information accompanies this paper at http://www.nature.com/ scientificreports

Competing financial interests: The authors declare no competing financial interests.

How to cite this article: Ahn, S. \& Lee, S.J. Optimum periodicity of repeated contractile actions applied in mass transport. Sci. Rep. 5, 7800; DOI:10.1038/srep07800 (2015).

This work is licensed under a Creative Commons Attribution 4.0 International License. The images or other third party material in this article are included in the article's Creative Commons license, unless indicated otherwise in the credit line; if the material is not included under the Creative Commons license, users will need to obtain permission from the license holder in order to reproduce the material. To view a copy of this license, visit http://creativecommons.org/licenses/by/4.0/ 